\title{
REDUCIBILITY AND IRREDUCIBILITY OF THE GAUSS-MANIN SYSTEM ASSOCIATED WITH A SELBERG TYPE INTEGRAL
}

\author{
KATSUHISA MIMACHI
}

\section{Dedicated to professor Joji Kajiwara on his 60th birthdy}

Consider the function with variables $z_{1}, \ldots, z_{m}$

$$
\int_{\Gamma} \prod_{1 \leq i \leq j \leq n}\left(t_{i}-t_{j}\right)^{\nu} \prod_{\substack{1 \leq i \leq n \\ 1 \leq j \leq m}}\left(t_{i}-z_{j}\right)^{\lambda_{j}} d t_{1} d t_{2} \ldots d t_{n},
$$

where $\nu$ and $\lambda_{j}(j=1, \ldots, m)$ are complex numbers and $\Gamma$ is a suitably chosen integral domain. In case $m=2$, if we set $\left[z_{1}, z_{2}\right]^{n}$ as $\Gamma$, it is the Selberg integral [22]. Our function can be regarded as an extention of it; so we may call (0.1) a Selberg type integral. It is known that (0.1) satisfies a Gauss-Manin system, i.e. a system of rationally holonomic differential equations [3], [21].

The purpose of the present paper is to study the (ir)reducibility conditions of the Gauss-Manin system associated with the integral above. Here the (ir)reducibility of a system means the (ir)reducibility of its monodromy representation.

Throughout our arguments, we adopt a framework of the theory of twisted rational de Rham cohomology and twisted homology, which will be briefly reviewed in Section 1. Let the many-valued $n$-form $\Phi d t_{1} \ldots d t_{n}$ be the integrand of (0.1). A basis of the twisted $n$-th de Rham cohomology group attached to $\Phi$ naturally induces a system of matrix valued differential equations of the first order, which is called the Gauss-Manin system. Our first result is its explicit expression (Proposition 2.1). Luckily enough, our system happens to have logarithmic poles with residues of constant matrices, which makes our discussion much simpler. A study about (ir)reducibility is made in Section 3. Our second result is the irreducibility condition when $\nu=0$. In this case, it is shown that if $\lambda_{j}(1 \leq j \leq m) \notin \mathbf{Z}$ and $\sum_{j=1}^{m} \lambda_{j} \notin \mathbf{Z}$ then our system is irreducible (Theorem 3.1). This suggests that

Received May 7, 1993. 
our Gauss-Manin system is generically irreducible, though we could not succeed in finding (ir)reducibility conditions in general setting. If a given system of differential equations has a subsystem, then the monodromy representation of the original one is reducible, of course. It seems an interesting problem to find a subsystem of our system. We find four reducible cases, when $m=3$ (Theorem 3.3). We also prove that the Lie algebra generated by the residue matrices is isomorphic to the general linear Lie algebra $\mathfrak{g l}(n+1 ; \mathbf{C})$ (Proposition 3.4$)$. The author believes that this Lie algebra should shed light on the irreducibility conditions in the future. When $m$ is arbitrary, we obtain a reducibility condition and a corresponding subsystem in Theorem 3.5: We find that if $0 \leq \ell \leq n-1$ and $2 \lambda_{j}+\nu \ell=0(j=$ $1, \ldots, m-2)$ then our system is reducible. By virtue of the formula in Theorem 3.5 in the case $\ell=1$, we can derive a system of the differential equations of the second order which is satisfied by the integral (0.1) (Theorem 4.1). This system is related with the spherical functions of $\mathrm{BC}$ type defined in [11].

We finally give a comment that the integral $(0.1)$ is used to give an integral representation of the solution of $\mathbf{a l}(2)$ Knizhnik-Zamolodchikov equation in Conformal field theory. Its monodromy representation is studied in various aspects and related with our work. See [6], [8], [21], [26].

\section{Twisted cohomology and homology}

Let $z_{1}, \ldots, z_{m}$ be complex parameters. Let

$$
\Phi=\prod_{1 \leq i \leq j \leq n}\left(t_{i}-t_{j}\right)^{\nu} \prod_{\substack{1 \leq i \leq n \\ 1 \leq j \leq m}}\left(t_{i}-z_{j}\right)^{\lambda_{j}}
$$

be a many valued holomorphic function with variables $t_{1}, \ldots, t_{n}$ on $X=\mathbf{C}^{n} \backslash D$, where

$$
D:=\bigcup_{1 \leq i<j \leq n}\left\{t_{i}-t_{j}=0\right\} \underset{\substack{1 \leq i \leq n \\ 1 \leq j \leq m}}{\cup}\left\{t_{i}-z_{j}=0\right\},
$$

and $\nu$ and $\lambda_{j}(1 \leq j \leq m)$ complex numbers. The symmetric group $S_{n}$ acts freely on $X$ as permutations of the coordinates $t_{1}, \ldots, t_{n}$.

Let $\Omega^{p}(* D)$ be the space of rational $p$-forms on $\mathbf{C}^{n}$ that are holomorphic on $X$. Then the (single-valued holomorphic) 1 -form $\omega:=d \Phi / \Phi$ on $X$ determines a rationally integrable connection (Gauss-Manin connection)

$$
\nabla_{\omega}: \Omega^{p}(* D) \ni \varphi \mapsto \nabla_{\omega} \varphi=d \varphi+\omega \wedge \varphi \in \Omega^{p+1}(* D)
$$

Then we have the following complex: 


$$
0 \rightarrow \Omega^{0}(* D) \stackrel{\nabla_{\omega}}{\rightarrow} \Omega^{1}(* D) \stackrel{\nabla_{\omega}}{\rightarrow} \cdots \stackrel{\nabla_{\omega}}{\rightarrow} \Omega^{n}(* D) \rightarrow 0 .
$$

Its $p$-th cohomology, denoted by $H^{p}\left(\Omega^{*}(* D), \nabla_{\omega}\right)$, is called the $p$-th twisted rational de Rham cohomology associated with the connection form $\omega$. The following fact is fundamental.

Theorem 1.1 ([3], [9]). (1) Suppose that

(Mon): non of the values of $i\left(\lambda_{j}+\frac{1}{2}(i-1) \nu\right)(1 \leq j \leq m, 1 \leq i \leq n)$, $\frac{1}{2} i(i+1) \nu(1 \leq i \leq n),(i-1-n) \sum_{k=1}^{m} \lambda_{k}+\left\{\left(\begin{array}{c}i-1 \\ 2\end{array}\right)+\left(\begin{array}{c}n \\ 2\end{array}\right)\right\} \nu(1 \leq i \leq n)$ lies in $\mathbf{N} \backslash\{0\}$.

Then the symmetric part $H^{n}\left(\Omega^{*}(* D), \nabla_{\omega}\right)^{s_{n}}$ of the cohomology $H^{n}\left(\Omega^{*}(* D)\right.$, $\nabla_{\omega}$ ) is generated by the symmetrization of the logarithmic forms

$$
d \log \left(t_{1}-z_{i_{1}}\right) \wedge d \log \left(t_{2}-z_{i_{2}}\right) \wedge \cdots \wedge d \log \left(t_{n}-z_{i_{n}}\right)
$$

for $1 \leq i_{1}, \ldots, i_{n} \leq m$.

(2) If we suppose $\prod_{0 \leq k \leq n-1}\left(\lambda_{m}+\frac{\nu}{2} k\right) \neq 0$, in addition to the assumption (Mon), then a basis of $H^{n}\left(\Omega^{*}(* D), \nabla_{\omega}\right)^{S_{n}}$ is given by the symmetrization of

$$
d \log \left(t_{1}-z_{i_{1}}\right) \wedge d \log \left(t_{2}-z_{i_{2}}\right) \wedge \cdots \wedge d \log \left(t_{n}-z_{i_{n}}\right)
$$

for $1 \leq i_{1}, \ldots, i_{n} \leq m-1$.

Then the rank of $H^{n}\left(\Omega^{*}(* D), \nabla_{\omega}\right)^{s_{n}}$ is $\left(\begin{array}{c}n+m-2 \\ m-2\end{array}\right)$.

This theorem was first proved by Aomoto [3]. His proof depends on the arguments for the generalized Pochhammer differential equations. On the other hand, Esnault-Schechtman-Viehweg gave another proof of (1) under more general setting (They treat the case of arbitrary hyperplane arrangements [9].). Their proof is given by the combination of the arguments for the degeneracy of the HodgeDeligne spectral sequence and a result by Brieskorn [5].

Let $S_{\omega}$ be the local system on $X$ defined by the monodromy of $\Phi$ and $S_{\omega}^{*}$ the dual local system of $S_{\omega}$. Let $H_{p}\left(X, S_{\omega}^{*}\right)$ be the $p$-th homology group with coefficients in $S_{\omega}^{*}$. Then we have the perfect pairing (see [7], [23])

$$
\begin{gathered}
H_{p}\left(X, S_{\omega}^{*}\right) \times H^{p}\left(\Omega^{\cdot}(* D), \nabla_{\omega}\right) \rightarrow \mathbf{C}, \\
(\Gamma, \varphi) \mapsto \int_{\Gamma} \varphi \Phi d t_{1} \ldots, d t_{n},
\end{gathered}
$$


which induces the pairing of the symmetric parts:

$$
H_{p}\left(X, S_{\omega}^{*}\right)^{S_{n}} \times H^{p}\left(\Omega^{*}(* D), \nabla_{\omega}\right)^{S_{n}} \rightarrow \mathbf{C} .
$$

In this paper we consider only the $\left(S_{n^{-}}\right)$symmetric (co)homology, i.e., the (twisted) cycles $\Gamma$ in the integral $(0.1)$ are so chosen that

$$
\int_{\Gamma} \varphi\left(\Phi d t_{1} \ldots, d t_{n}\right)^{\sigma}=\int_{\Gamma} \varphi \Phi d t_{1} \ldots, d t_{n}
$$

for all symmetric rational functions $\varphi \in \mathbf{C}\left(t_{1}, \ldots, t_{n}\right)$ and $\sigma \in S_{n}$.

We refer the reader to [1], [14], [15], [16], [21], [25] for related works with the twisted cohomology and homology.

By using this framework, in the next section, we derive a Gauss-Manin system associated with this Selberg type integral.

\section{Gauss-Manin system}

This section is devoted to deriving a system of matrix valued differential equations of the first order associated with the Selberg type integral

$$
\int_{\Gamma} \prod_{1 \leq i \leq j \leq n}\left(t_{i}-t_{j}\right)^{\nu} \prod_{\substack{1 \leq i \leq n \\ 1 \leq j \leq m}}\left(t_{i}-z_{j}\right)^{\lambda_{j}} d t_{1} d t_{2} \ldots d t_{n}
$$

with variables $z_{1}, \ldots, z_{m}$. In what follows we suppose $m \geq 2$ and fix a cycle $\Gamma$ satisfying (1.4).

Notice that for a rational function $\varphi$, we have

$$
\frac{\partial}{\partial z_{s}} \int_{\Gamma} \varphi \Phi d t_{1} d t_{2} \ldots d t_{n}=\int_{\Gamma}\left(\nabla_{z_{s}} \varphi\right) \Phi d t_{1} d t_{2} \ldots d t_{n}
$$

where

$$
\nabla_{z_{s}}:=\frac{\partial}{\partial z_{s}}+\frac{\partial \log \Phi}{\partial z_{s}}
$$

For brevity we put

$$
\varphi_{\left(a_{1}, \ldots, a_{n}\right)}:=\prod_{1 \leq i \leq n}\left(t_{i}-z_{a_{i}}\right)^{-1}
$$

for $1 \leq a_{i} \leq m(1 \leq i \leq n)$ and also use the notation

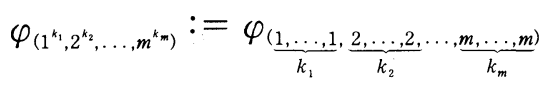


for $k_{1}, \ldots, k_{m}$ such that $k_{i} \geq 0(1 \leq i \leq m)$ and $k_{1}+\cdots+k_{m}=n$.

Moreover we write

$$
\langle\varphi\rangle:=\int_{\Gamma} \varphi \Phi d t_{1} d t_{2} \ldots d t_{n}
$$

for all symmetric twisted cycles $\Gamma$, and $\varphi_{1} \equiv \varphi_{2}$ for $\left\langle\varphi_{1}\right\rangle=\left\langle\varphi_{2}\right\rangle$.

Under these notations, we derive a system of differential equations of the first order (Gauss-Manin system) with respect to the basis

$$
\left\{\left\langle\varphi_{\left(1^{k_{1}}, 2^{k_{2}}, \ldots,(m-1)^{k_{m-1}}\right)}\right\rangle ; k_{i} \geq 0(1 \leq i \leq m-1), \sum_{1 \leq i \leq m-1} k_{i}=n\right\}
$$

Note that the cardinality of the set (2.3) is $\left(\begin{array}{c}n+m-2 \\ m-2\end{array}\right)$.

Proposition 2.1. (i) For $r=1, \ldots, m-1$, we have

$$
\begin{aligned}
& \frac{\partial}{\partial z_{r}}\left\langle\varphi_{\left(1^{k_{1}}, 2^{k_{2}}, \ldots,(m-1)^{\left.k_{m-1}\right)}\right.}\right\rangle= \\
& -\left(\lambda_{r}+\frac{\nu}{2} k_{r}\right) \sum_{\substack{1 \leq s \leq m-1 \\
s \neq r}} \frac{k_{s}}{z_{r}-z_{s}}\left\langle\varphi_{\left(\ldots, r^{k, 1}, \ldots, s^{k,-1}, \ldots\right)}\right\rangle \\
& +\left[\sum_{\substack{1 \leq s \leq m-1 \\
s \neq r}} \frac{k_{r} k_{s} \nu+\lambda_{s} k_{r}+\lambda_{r} k_{s}}{z_{r}-z_{s}}+\frac{k_{r}\left\{\lambda_{r}+\lambda_{m}+\frac{\nu}{2}\left(k_{r}-1\right)\right\}}{z_{r}-z_{m}}\right] \\
& \times\left\langle\varphi_{\left(1^{k_{1}}, \ldots,(m-1)^{k_{m-1}}\right)}\right\rangle \\
& +\sum_{\substack{1 \leq s \leq m-1 \\
s \neq r}} k_{r}\left(\lambda_{s}+\frac{\nu}{2} k_{s}\right)\left\{\frac{1}{z_{r}-z_{m}}-\frac{1}{z_{r}-z_{s}}\right\} \\
& \times\left\langle\varphi_{\left(\ldots, r^{k,-1}, \ldots, s^{k,+1}, \ldots\right)}\right\rangle,
\end{aligned}
$$

and

$$
\begin{aligned}
& \frac{\partial}{\partial z_{m}}\left\langle\varphi_{\left(1^{k_{1}}, 2^{k_{2}}, \ldots,(m-1)^{\left.k_{m-1}\right)}\right.}\right\rangle= \\
& \sum_{1 \leq s \leq m-1} \frac{k_{s}\left\{\lambda_{m}+\lambda_{s}+\frac{\nu}{2}\left(k_{s}-1\right)\right\}}{z_{m}-z_{s}}\left\langle\varphi_{\left(1^{k_{1}}, \ldots,(m-1)^{k_{m-1}}\right)}\right\rangle \\
& +\sum_{1 \leq s \leq m-1} \sum_{\substack{1 \leq r \leq m-1 \\
r \neq s}} \frac{k_{s}\left(\lambda_{r}+\frac{\nu}{2} k_{r}\right)}{z_{m}-z_{s}}\left\langle\varphi_{\left(\ldots, s^{k,-1}, \ldots, r^{k_{r}+1}, \ldots\right)}\right\rangle .
\end{aligned}
$$


Proof. First we derive the equality (2.7) used below. By the Stokes theorem, we have

$$
0 \equiv \frac{\nu}{2} \sum_{1 \leq j \leq n-1} \varphi_{\left(a_{1}, \ldots, a_{n-1}, a_{j}\right)}+\sum_{1 \leq a \leq m} \lambda_{a} \varphi_{\left(a_{1}, \ldots, a_{n-1}, a\right)}
$$

indeed, the right hand side of (2.6) is

$$
\begin{aligned}
\sum_{j \neq n)} \frac{\nu}{2}\left[\frac{1}{t_{n}-t_{j}} \frac{1}{t_{j}-z_{a_{i}}}+\frac{1}{t_{j}-t_{n}} \frac{1}{t_{n}-z_{a_{j}}}\right] \sum_{\substack{1 \leq i \leq n-1 \\
i \neq j}}\left(t_{i}-z_{a_{i}}\right)^{-1} \\
\quad+\prod_{1 \leq i \leq n-1}\left(t_{i}-z_{a_{i}}\right)^{-1} \sum_{1 \leq a \leq m} \lambda_{a}\left(t_{n}-z_{a}\right)^{-1} \\
\equiv\left[\sum_{j(\neq n)} \frac{\nu}{t_{n}-t_{j}}+\sum_{1 \leq a \leq m} \frac{\lambda_{a}}{t_{n}-t_{a}}\right] \prod_{1 \leq i \leq n-1}\left(t_{i}-z_{a_{i}}\right)^{-1} \\
=\nabla_{t_{n}} \prod_{1 \leq i \leq n-1}\left(t_{i}-z_{a_{i}}\right)^{-1} \equiv 0 .
\end{aligned}
$$

Set

$$
\begin{aligned}
& a_{1}=\cdots=a_{k_{1}-1}=1 \\
& a_{k_{1}+\cdots+k_{s-1}}=\cdots=a_{k_{1}+\cdots+k_{s}-1}=s \text { for } 2 \leq s \leq m-1
\end{aligned}
$$

and $a_{n}=m$ in the relation (2.6). Then the equality

$$
\begin{aligned}
0 \equiv & \left\{\lambda_{1}+\frac{\nu}{2}\left(k_{1}-1\right)\right\} \varphi_{\left(1^{k_{1}}, \ldots,(m-1)^{\left.k_{m-1}\right)}\right.} \\
& +\sum_{2 \leq s \leq m-1}\left(\lambda_{s}+\frac{\nu}{2} k_{s}\right) \varphi_{\left(1^{k_{1}-1}, \ldots, s^{k_{s}+1}, \ldots\right)} \\
& +\lambda_{m} \varphi_{\left(1^{k_{1}-1}, \ldots,(m-1)^{\left.k_{m-1}, m^{1}\right)}\right.}
\end{aligned}
$$

holds.

By the help of the equality (2.7), we derive the equations (2.4) and (2.5).

Direct calculation shows

$$
\begin{aligned}
& =\left[\sum_{1 \leq i \leq k_{1}}\left\{-\nabla_{t_{i}}+\sum_{j(\neq i)} \frac{\nu}{t_{i}-t_{j}}+\sum_{1 \leq s \leq m} \frac{\lambda_{s}}{t_{i}-z_{s}}\right\}+\frac{\partial \log \Phi}{\partial z_{1}}\right] \\
& \quad \times \varphi_{\left(1^{k_{1}}, \ldots,(m-1)^{\left.k_{m-1}\right)}\right.} \\
& \left.\equiv \sum_{1 \leq i \leq k_{1}}\left\{\sum_{j(\neq i)} \frac{\nu}{t_{i}-t_{j}}+\sum_{2 \leq s \leq m} \frac{\lambda_{s}}{t_{i}-z_{s}}\right\}+\sum_{k_{1}+1 \leq i \leq n} \frac{-\lambda_{1}}{t_{i}-z_{1}}\right]
\end{aligned}
$$


GAUSS-MANIN SYSTEM WITH SELBERG TYPE

49

$$
\begin{aligned}
& \times \varphi_{\left(1^{k_{1}}, \ldots,(m-1)^{\left.k_{m-1}\right)}\right.} \\
& \equiv\left[k_{1}\left\{\sum_{k_{1}+1 \leq j \leq n} \frac{\nu}{t_{1}-t_{j}}+\sum_{2 \leq s \leq m} \frac{\lambda_{s}}{t_{1}-z_{s}}\right\}-\sum_{k_{1}+1 \leq i \leq n} \frac{\lambda_{1}}{t_{i}-z_{1}}\right] \\
& \quad \times \varphi_{\left(1^{k_{1}}, \ldots,(m-1)^{\left.k_{m-1}\right)}\right.} .
\end{aligned}
$$

By virtue of the equality

$$
\begin{aligned}
& \frac{1}{t_{1}-t_{j}} \frac{1}{t_{1}-z_{1}} \frac{1}{t_{j}-z_{s}}+\frac{1}{t_{j}-t_{1}} \frac{1}{t_{j}-z_{1}} \frac{1}{t_{1}-z_{s}} \\
= & \frac{-1}{z_{1}-z_{s}}\left[\frac{1}{t_{1}-z_{1}}-\frac{1}{t_{1}-z_{s}}\right]\left[\frac{1}{t_{j}-z_{1}}-\frac{1}{t_{j}-z_{s}}\right],
\end{aligned}
$$

we have

$$
\begin{gathered}
\sum_{k_{1}+1 \leq j \leq n} \frac{\nu}{t_{1}-t_{j}} \varphi_{\left(1^{k_{1}}, \ldots,(m-1)^{k_{m-1}}\right)} \\
=-\sum_{2 \leq s \leq m-1} \frac{k_{s}}{2\left(z_{1}-z_{s}\right)}\left\{\varphi_{\left(1^{k_{1}+1}, \ldots, s^{k_{s}-1}, \ldots\right)}-2 \varphi_{\left(1^{k_{1}}, \ldots,(m-1)^{k_{m-1}}\right)}+\varphi_{\left(1^{k_{1}-1}, \ldots, s^{k_{s}+1}, \ldots\right)}\right\} .
\end{gathered}
$$

The equality

$$
\frac{1}{t_{i}-z_{1}} \frac{1}{t_{i}-z_{s}}=\frac{1}{z_{1}-z_{s}}\left[\frac{1}{t_{i}-z_{1}}-\frac{1}{t_{i}-z_{s}}\right]
$$

and (2.7) lead to

$$
\begin{aligned}
& \sum_{2 \leq s \leq m} \frac{\lambda_{s}}{t_{1}-z_{s}} \varphi_{\left(1^{k_{1}}, \ldots,(m-1)^{\left.k_{m-1}\right)}\right.} \\
& =\sum_{2 \leq s \leq m-1} \frac{\lambda_{s}}{z_{1}-z_{s}}\left\{\varphi_{\left(1^{k_{1}}, \ldots,(m-1)^{\left.k_{m-1}\right)}\right.}-\varphi_{\left(1^{k_{1}-1}, \ldots, s^{k_{s}+1}, \ldots\right)}\right\} \\
& +\frac{\lambda_{m}}{z_{1}-z_{m}}\left\{\varphi_{\left(1^{k_{1}}, \ldots,(m-1)^{\left.k_{m-1}\right)}\right.}-\varphi_{\left(1^{k_{1}-1}, \ldots,(m-1)^{\left.k_{m-1}, m^{\prime}\right)}\right.}\right\} \\
& \equiv \sum_{2 \leq s \leq m-1} \frac{\lambda_{s}}{z_{1}-z_{s}}\left\{\varphi_{\left(1^{k_{1}}, \ldots,(m-1)^{\left.k_{m-1}\right)}\right.}-\varphi_{\left(1^{k_{1}-1}, \ldots, s^{k_{s}+1}, \ldots\right)}\right\} \\
& +\frac{\lambda_{m}+\lambda_{1}+\frac{\nu}{2}\left(k_{1}-1\right)}{z_{1}-z_{m}} \varphi_{\left(1^{k_{1}}, \ldots,(m-1)^{\left.k_{m-1}\right)}\right.} \\
& +\sum_{2 \leq s \leq m-1} \frac{\lambda_{s}+\frac{\nu}{2} k_{s}}{z_{1}-z_{m}} \varphi_{\left(1^{k_{1}-1}, \ldots, s^{k_{s}+1}, \ldots\right)} .
\end{aligned}
$$

https://doi.org/10.1017/S0027763000004633 Published online by Cambridge University Press 
Similarly, the equality (2.10) gives

$$
\begin{gathered}
\sum_{k_{1}+1 \leq i \leq n} \frac{1}{t_{i}-z_{1}} \varphi_{\left(1^{k_{1}}, \ldots,(m-1)^{k_{m-1}}\right)} \\
=\sum_{2 \leq s \leq m-1} \frac{k_{s}}{z_{s}-z_{1}}\left\{\varphi_{\left(1^{k_{1}}, \ldots,(m-1)^{\left.k_{m-1}\right)}\right.}-\varphi_{\left(1^{k_{1}+1}, \ldots, s^{k_{s}-1}, \ldots\right)}\right\} .
\end{gathered}
$$

Therefore we get the differential equation (2.4) with $r=1$ by substituting (2.9), (2.11) and (2.12) into (2.8). The equations with respect to $\nabla_{z_{r}}(2 \leq r \leq$ $m-1)$ are obtained by changing the suffices accordingly.

The equation for $\partial / \partial z_{m}$ is given by the same way as follows. First we note the equality

$$
\begin{aligned}
0 \equiv & \left\{\lambda_{s}+\frac{\nu}{2}\left(k_{s}-1\right)\right\} \varphi_{\left(1^{k_{1}}, \ldots,(m-1)^{k_{m-1}}\right)} \\
& +\sum_{\substack{1 \leq t \leq m-1 \\
t \neq s}}\left(\lambda_{t}+\frac{\nu}{2} k_{t}\right) \varphi_{\left(\ldots, s^{k^{s}-1}, \ldots, t^{k^{*+1}}, \ldots\right)} \\
& +\lambda_{m} \varphi_{\left(\ldots, s^{k_{s-1}}, \ldots,(m-1)^{\left.k_{m-1}, m^{\prime}\right)}\right.}
\end{aligned}
$$

for $1 \leq s \leq m-1$, which is given by changing the suffices in (2.6) appropriately. By (2.10) and (2.13), we have

$$
\begin{aligned}
& \nabla_{z_{m}} \varphi_{\left(1^{k_{1}}, \ldots,(m-1)^{\left.k_{m-1}\right)}\right.} \\
& \equiv \sum_{1 \leq i \leq n} \frac{\lambda_{m}}{z_{m}-t_{i}} \varphi_{\left(1^{k_{1}}, \ldots,(m-1)^{\left.k_{m-1}\right)}\right.} \\
& =\sum_{1 \leq s \leq m-1} \frac{\lambda_{m} k_{s}}{z_{m}-z_{s}}\left\{\varphi_{\left(1^{k_{1}}, \ldots,(m-1)^{\left.k_{m-1}\right)}\right.}-\varphi_{\left(\ldots, s^{k,-1}, \ldots, m^{1}\right)}\right\} \\
& \equiv \sum_{1 \leq s \leq m-1} \frac{k_{s}}{z_{m}-z_{s}}\left\{\left\{\lambda_{m}+\lambda_{s}+\frac{\nu}{2}\left(k_{s}-1\right)\right\} \varphi_{\left(1^{k_{1}}, \ldots,(m-1)^{k_{m-1}}\right)}\right. \\
& \left.+\sum_{\substack{1 \leq t \leq m-1 \\
t \neq s}}\left(\lambda_{t}+\frac{\nu}{2} k_{t}\right) \varphi_{\left(\ldots s^{s_{s}-1}, \ldots, t^{k^{t}+1}, \ldots\right)}\right\} \text {, }
\end{aligned}
$$

which is (2.5).

Remark 2.2. To derive the system (2.4) and (2.5), we need no conditions.

Remark 2.3. Our system can be written as

$$
d \Psi=\left\{\sum_{1 \leq i<j \leq m} A_{i j} d \log \left(z_{i}-z_{j}\right)\right\} \Psi .
$$


Here $A_{i j}, 1 \leq i<j \leq m$, are square matrices with size $\left(\begin{array}{c}n+m-2 \\ m-2\end{array}\right)$ and with C coefficients, and $\Psi={ }^{t}\left(\left\langle\varphi_{\left(1^{n}\right)}\right\rangle, \ldots,\left\langle\varphi_{\left((m-1)^{n}\right)}\right\rangle\right)$. It is known $([18])$ that $(2.15)$ is integrable if and only if the following conditions are satisfied.

$$
\begin{gathered}
{\left[A_{i j}, A_{i k}+A_{j k}\right]=\left[A_{i j}+A_{i k}, A_{j k}\right]=0 \text { for } i<j<k,} \\
{\left[A_{i j}, A_{k \ell}\right]=0 \text { for distinct } i, j, k, \ell .}
\end{gathered}
$$

These relations are called the infinitesimal pure braid relations, and also called the classical Yang-Baxter relations, which play an important role in Mathematical Physics. The linear independence of the basis

$$
\left\{\left\langle\varphi_{\left(1^{k_{1}}, 2^{k_{2}}, \ldots,(m-1)^{k_{m-1}}\right)}\right\rangle ; k_{i} \geq 0, \sum_{1 \leq i \leq m-1} k_{i}=n\right\}
$$

guarantees the integrability of the system. Once we get the integrability under the conditions (Mon), it is guaranteed without any conditions, since the elements of $A_{i j}$ are linear in $\lambda_{j}(j=1, \ldots, m)$ and $\nu$.

\section{Reducibility and irreducibility}

In this section, we study the (ir)reducibility of the Gauss-Manin system in Proposition 2.1.

Theorem 3.1. When $\nu=0$, the Gauss-Manin system (2.4-5) is irreducible if $\lambda_{i}$ $\notin \mathbf{Z}_{(1 \leq i \leq m)}$ and $\sum_{i=1}^{m} \lambda_{i} \notin \mathbf{Z}$.

Proof. Notice that the integral (0.1) reduces to the product of integrals of Pochhammer type (i.e. case $n=1$ )

$$
\int \prod_{\substack{1 \leq i \leq n \\ 1 \leq j \leq m}}\left(t_{i}-z_{j}\right)^{\lambda_{j}} d t_{1} d t_{2} \ldots d t_{n}=\prod_{1 \leq i \leq n} \int \prod_{1 \leq j \leq m}\left(t_{i}-z_{j}\right)^{\lambda_{j}} d t_{i} .
$$

Takano-Bannai [24] showed that when $n=1$, if $\lambda_{i} \notin \mathbf{Z}$ and $\sum_{i=1}^{m} \lambda_{i} \notin \mathbf{Z}$ then the monodromy representation is irreducible. On the other hand T. Sasaki [20] showed that the Zariski closure of this monodromy group is the general linear group $G L(m-1 ; \mathbf{C})$. Hence there exists a basis $B_{1}$ of the homology $H_{1}\left(X, S_{\omega}^{*}\right)$ in the $n=1$ case which gives a fundamental representation of $G L(m-1 ; \mathbf{C})$. On the other hand, each element in the homology $H_{n}\left(X, S_{\omega}^{*}\right)^{S_{n}}$ corresponds to the $n$-fold symmetric tensor of elements in $B_{1}$. Thus by Weyl's reciprocity, there exists a basis of $H_{n}\left(X, S_{\omega}^{*}\right)^{S_{n}}$ which gives an irreducible representation of $G L(m-1 ; \mathbf{C})$, 
and consequently of the monodromy representation of (2.4-5).

Theorem 3.1 suggests us that under some generic condition for $\nu$ and $\lambda_{i}$ Gauss-Manin system (2.4-5) is irreducibile, although we could not prove it generally.

We consider the case $m=3$, for a while.

Thanks to Proposition 2.1, the Gauss-Manin system takes the form

$$
d \Psi=\left\{\sum_{1 \leq i<j \leq 3} A_{i j} d \log \left(z_{i}-z_{j}\right)\right\} \Psi
$$

with respect to the basis $\Psi={ }^{t}\left(\left\langle\varphi_{\left(1^{n}\right)}\right\rangle,\left\langle\varphi_{\left(1^{n-1}, 2^{1}\right)}\right\rangle, \ldots,\left\langle\varphi_{\left(2^{n}\right)}\right\rangle\right)$. Here the matrices $A_{i j}=\left(a_{s t}^{(i j)}\right)_{1 \leq s, t \leq n+1}$ do not depend on the $z_{j}$ 's and are given by

$$
\left\{\begin{array}{l}
a_{i+1, i}^{(12)}=-i\left(\lambda_{1}+\frac{\nu}{2}(n-i)\right), \\
a_{i, i+1}^{(12)}=-(n-i+1)\left(\lambda_{2}+\frac{\nu}{2}(i-1)\right), \\
a_{i, i}^{(12)}=(i-1)\left((n-i+1) \nu+(n-i+1) \lambda_{2}+(i-1) \lambda_{1}\right), \\
a_{i j}^{(12)}=0 \text { otherwise }
\end{array}\right.
$$

and

$$
\left\{\begin{array}{l}
a_{i, i}^{(13)}=(n-i+1)\left(\lambda_{1}+\lambda_{3}+\frac{\nu}{2}(n-i)\right), \\
a_{i, i+1}^{(13)}=(n-i+1)\left(\lambda_{2}+\frac{\nu}{2}(i-1)\right) \\
a_{i j}^{(13)}=0 \text { otherwise }
\end{array}\right.
$$

and

$$
\left\{\begin{array}{l}
a_{i+1, i}^{(23)}=i\left(\lambda_{1}+\frac{\nu}{2}(n-i)\right) \\
a_{i+1, i+1}^{(23)}=i\left(\lambda_{2}+\lambda_{3}+\frac{\nu}{2}(i-1)\right) \\
a_{i j}^{(23)}=0 \text { otherwise. }
\end{array}\right.
$$

These show the following: Fix an integer $k$ such that $0 \leq k \leq n-1$. If $\lambda_{1}+\frac{\nu}{2} k=0$, we have $a_{n-k+1, n-k}^{(12)}=a_{n-k+1, n-k}^{(13)}=a_{n-k+1, n-k}^{(23)}=0$. This yields a subsystem of the system (3.1) with a basis

$$
\left\{\varphi_{\left(1^{k_{1}}, 2^{k_{2}}\right)} ; 0 \leq k_{1} \leq k, k_{1}+k_{2}=n\right\} .
$$

Similarly for each $k$ such that $0 \leq k \leq n-1$, if $\lambda_{2}+\frac{\nu}{2} k=0$, we have a subsystem with a basis

$$
\left\{\varphi_{\left(1^{k_{1}}, 2^{k_{2}}\right)} ; 0 \leq k_{2} \leq k, k_{1}+k_{2}=n\right\} .
$$


The following question naturally arises: Are there other subsystems of (3.1)? To answer this question, we transform $A_{i j}$ by the triangular matrix $P_{13}$. Consider a matrix $P_{13}=\left(p_{i j}^{(13)}\right)_{1 \leq i, j \leq n+1}$ defined by

$$
\left\{\begin{array}{l}
p_{1 j}^{(13)}=1 \text { for } 1 \leq i \leq n+1 \\
p_{i j}^{(13)}=\prod_{t=0}^{i-2} \frac{\rho_{j}^{(13)}-\rho_{t+1}^{(13)}}{\left\{(n-t)\left(\lambda_{2}+\frac{\nu}{2} t\right)\right\}} \text { for } 2 \leq i \leq j \leq n+1, \\
p_{i j}^{(13)}=0, \text { otherwise, }
\end{array}\right.
$$

where $\rho_{i}^{(13)}=(n-i+1)\left(\lambda_{1}+\lambda_{3}+\frac{\nu}{2}(n-i)\right)$. Then we have the key lemma.

\section{LEMma 3.2. Under the condition}

$$
\prod_{t=0}^{n-1}\left(\lambda_{2}+\frac{\nu}{2} t\right) \prod_{k=0}^{2 n-2}\left(2 \lambda_{1}+2 \lambda_{3}+k \nu\right) \neq 0,
$$

we have

$$
P_{13}^{-1} A_{13} P_{13}=\operatorname{diag}\left(\rho_{1}^{(13)}, \rho_{2}^{(13)}, \ldots, \rho_{n+1}^{(13)}\right)
$$

and

$$
P_{13}^{-1} A_{23} P_{13}=B_{23}=\left(b_{i j}^{(23)}\right)_{1 \leq i, j \leq n+1}
$$

where

$$
\left\{\begin{aligned}
\frac{b_{i, i+1}^{(23)}=}{\left(\lambda_{1}+\lambda_{3}+\frac{\nu}{2}(n-i-1)\right)\left(\lambda_{3}+\frac{\nu}{2}(n-i)\right)\left(\lambda_{1}+\lambda_{2}+\lambda_{3}+\frac{\nu}{2}(2 n-i-1)\right)} & \left(\lambda_{1}+\lambda_{3}+(n-i) \nu\right)\left(\lambda_{1}+\lambda_{3}+\frac{\nu}{2}(2 n-2 i-1)\right) \\
b_{i+1, i}^{(23)}=- & (n-i+1) \\
& \quad \frac{\left(\lambda_{1}+\frac{\nu}{2}(n-i)\right)\left(\lambda_{2}+\frac{\nu}{2}(i-1)\right)\left(\lambda_{1}+\lambda_{3}+\frac{\nu}{2}(2 n-i)\right)}{\left(\lambda_{1}+\lambda_{3}+(n-i) \nu\right)\left(\lambda_{1}+\lambda_{3}+\frac{\nu}{2}(2 n-2 i+1)\right)} \\
b_{i, i}^{(23)}=- & b_{i+1, i}^{(23)}-b_{i-1, i}^{(23)}, \\
b_{i j}^{(23)}=0 & \text { otherwise. }
\end{aligned}\right.
$$


Proof. Since

$$
\begin{gathered}
\operatorname{det} P_{13}=\prod_{i=2}^{n+1} \prod_{t=0}^{i-2} \frac{\rho_{j}^{(13)}-\rho_{t+1}^{(13)}}{\left\{(n-t)\left(\lambda_{2}+\frac{\nu}{2} t\right)\right\}} \\
=\prod_{i=2}^{n+1} \prod_{t=0}^{i-2} \frac{(i-t-1)\left\{(i+t-2 n) \nu-2 \lambda_{1}-2 \lambda_{3}\right\}}{2(n-t)\left(\lambda_{2}+\frac{\nu}{2} t\right)}
\end{gathered}
$$

the condition $\operatorname{det} P_{13} \neq 0$ is equivalent to (3.6). Then it suffices to prove the equality $A_{23} P_{13}=P_{13} B_{23}$. The $(i, j)$-entry in this equality is

$$
\begin{aligned}
& a_{i, i-1}^{(23)} p_{i-1, j}^{(13)}+a_{i, i}^{(23)} p_{i j}^{(13)} \\
= & p_{i, j-1}^{(13)} b_{j-1, j}^{(23)}+p_{i, j}^{(13)} b_{j, j}^{(23)}+p_{i, j+1}^{(13)} b_{j+1, j}^{(23)} .
\end{aligned}
$$

Note that only the elements at $j=i-1, i, i+1, \ldots, n+1$ are not zero. At $j=i-1$, the above equality is obvious. Thus the only cases $j \geq i$ are crucial; so we suppose $j \geq i$.

First we get

$$
\begin{aligned}
a_{i, i-1}^{(23)} p_{i-1, j}^{(13)} & +a_{i, i}^{(23)} p_{i j}^{(13)} \\
=(i-1) & \prod_{t=0}^{i-2} \frac{\rho_{j}^{(13)}-\rho_{t+1}^{(13)}}{\left\{(n-t)\left(\lambda_{2}+\frac{\nu}{2} t\right)\right\}} \\
& \times\left[\frac{(n-i+2)\left(\lambda_{1}+\frac{\nu}{2}(n-i+1)\right)\left(\lambda_{2}+\frac{\nu}{2}(i-2)\right)}{(i-j-1)\left(\lambda_{1}+\lambda_{3}+\frac{\nu}{2}(2 n+2-i-j)\right)}\right. \\
& \left.+\left(\lambda_{2}+\lambda_{3}+\frac{\nu}{2}(i-2)\right)\right] .
\end{aligned}
$$

On the other hand, by noting

$$
\prod_{t=0}^{i-2}\left(\rho_{j-1}-\rho_{t+1}\right)=\frac{(i-j)\left(\lambda_{1}+\lambda_{3}+\frac{\nu}{2}(2 n-j+1)\right)}{(1-j)\left(\lambda_{1}+\lambda_{3}+\frac{\nu}{2}(2 n-i-j+2)\right)} \prod_{t=0}^{i-2}\left(\rho_{j}-\rho_{t+1}\right)
$$

and

$$
\prod_{t=0}^{i-2}\left(\rho_{j+1}-\rho_{t+1}\right)=\frac{-j\left(\lambda_{1}+\lambda_{3}+\frac{\nu}{2}(2 n-i-j+1)\right)}{(i-j-1)\left(\lambda_{1}+\lambda_{3}+\frac{\nu}{2}(2 n-j)\right)} \prod_{t=0}^{i-2}\left(\rho_{j}-\rho_{t+1}\right),
$$


we obtain

$$
\begin{aligned}
& p_{i, j-1}^{(13)} b_{j-1, j}^{(23)}+p_{i, j}^{(13)} b_{j, j}^{(23)}+p_{i, j+1}^{(13)} b_{j+1, j}^{(23)} \\
& =\prod_{t=0}^{i-2} \frac{\rho_{j}^{(13)}-\rho_{t+1}^{(13)}}{\left\{(n-t)\left(\lambda_{2}+\frac{\nu}{2} t\right)\right\}}\left[\frac{(i-j)\left(\lambda_{1}+\lambda_{3}+\frac{\nu}{2}(2 n-j+1)\right)}{(1-j)\left(\lambda_{1}+\lambda_{3}+\frac{\nu}{2}(2 n-i-j+2)\right)} b_{j-1, j}^{(23)}\right. \\
& \left.+\left(-b_{j-1, j}^{(23)}-b_{j+1, j}^{(23)}\right)+\frac{-j\left(\lambda_{1}+\lambda_{3}+\frac{\nu}{2}(2 n-i-j+1)\right)}{(i-j-1)\left(\lambda_{1}+\lambda_{3}+\frac{\nu}{2}(2 n-j)\right)} b_{j+1, j}^{(23)}\right] \\
& =\prod_{t=0}^{i-2} \frac{\rho_{j}^{(13)}-\rho_{t+1}^{(13)}}{\left\{(n-t)\left(\lambda_{2}+\frac{\nu}{2} t\right)\right\}}\left[\frac{(i-1)\left(\lambda_{1}+\lambda_{3}+\nu(n-j+1)\right)}{(1-j)\left(\lambda_{1}+\lambda_{3}+\frac{\nu}{2}(2 n-i-j+2)\right)} b_{j-1, j}^{(23)}\right. \\
& \left.-\frac{(i-1)\left(\lambda_{1}+\lambda_{3}+\nu(n-j)\right)}{(i-j-1)\left(\lambda_{1}+\lambda_{3}+\frac{\nu}{2}(2 n-j)\right)^{(23)}} b_{j+1, j}\right] \\
& =\prod_{t=0}^{i-2} \frac{\rho_{j}^{(13)}-\rho_{t+1}^{(13)}}{\left\{(n-t)\left(\lambda_{2}+\frac{\nu}{2} t\right)\right\}} \frac{i-1}{\left(\lambda_{1}+\lambda_{3}+\frac{\nu}{2}(2 n-2 j+1)\right)} \\
& \times\left[\frac{\left(\lambda_{1}+\lambda_{3}+\frac{\nu}{2}(n-j)\right)\left(\lambda_{3}+\frac{\nu}{2}(n-j+1)\right)\left(\lambda_{1}+\lambda_{2}+\lambda_{3}+\frac{\nu}{2}(2 n-j)\right)}{\lambda_{1}+\lambda_{3}+\frac{\nu}{2}(2 n-i-j+2)}\right. \\
& \left.+\frac{(n-j+1)\left(\lambda_{1}+\frac{\nu}{2}(n-j)\right)\left(\lambda_{2}+\frac{\nu}{2}(j-1)\right)}{i-j-1}\right]
\end{aligned}
$$

Therefore the equality $(3.9)$ reduces to

$$
\begin{aligned}
& \left(\lambda_{1}+\lambda_{3}+\frac{\nu}{2}(2 n-2 j+1)\right)\left\{(n-i+2)\left(\lambda_{1}+\frac{\nu}{2}(n-i+1)\right)\left(\lambda_{2}+\frac{\nu}{2}(i-2)\right)\right. \\
& \left.\quad+(i-j-1)\left(\lambda_{1}+\lambda_{3}+\frac{\nu}{2}(2 n-i-j+2)\right)\left(\lambda_{2}+\lambda_{3}+\frac{\nu}{2}(i-2)\right)\right\} \\
& =(i-j-1)\left(\lambda_{1}+\lambda_{3}+\frac{\nu}{2}(n-j)\right)\left(\lambda_{3}+\frac{\nu}{2}(n-j+1)\right)\left(\lambda_{1}+\lambda_{2}+\lambda_{3}+\frac{\nu}{2}(2 n-j)\right) \\
& +(n-j+1)\left(\lambda_{1}+\lambda_{3}+\frac{\nu}{2}(2 n-i-j+2)\right)\left(\lambda_{1}+\frac{\nu}{2}(n-j)\right)\left(\lambda_{2}+\frac{\nu}{2}(j-1)\right),
\end{aligned}
$$


which can be checked readily. This completes the proof.

Notice that $P_{13}^{-1} A_{13} P_{13}$ is diagonal and $P_{13}^{-1} A_{23} P_{13}$ is tri-diagonal.

THEOREM 3.3. If one of the followings holds, the Gauss-Manin connection (3.1) is reducible:

(1) $\lambda_{1}+\frac{\nu}{2} k=0$ for some $k$ such that $0 \leq k \leq n-1$,

(2) $\lambda_{2}+\frac{\nu}{2} k=0$ for some $k$ such that $0 \leq k \leq n-1$,

(3) under the condition (3.6),

$$
\lambda_{3}+\frac{\nu}{2} k=0 \text { for some } k \text { such that } 0 \leq k \leq n-1 \text {, }
$$

(4). under the condition (3.6),

$$
\lambda_{1}+\lambda_{2}+\lambda_{3}+\frac{\nu}{2}(2 n-k-2)=0 \text { for some } k \text { such that } 0 \leq k \leq n-1 .
$$

Proof. The assertions (1), (2) have already been proved. The assertions (3) and (4) are derived from Lemma 3.2, since one of the off-diagonal elements of $P_{13}^{-1} A_{23} P_{13}$ vanishes.

On the other hand, as a by-product of the argument above, we have

Proposition 3.4. Under the condition (3.6), the Lie algebra Lie $\left(A_{12}, A_{13}\right.$, $A_{23}$ ) generated by three matrices $A_{12}, A_{13}$ and $A_{23}$ is isomorphic to the general linear Lie algebra $\mathfrak{g l}(n+1 ; \mathbf{C})$.

Proof. It suffices to show that Lie algebra generated by two matrices $P_{13}^{-1} A_{13} P_{13}$ and $P_{13}^{-1} A_{23} P_{13}$ is isomorphic to $\mathrm{gl}(n+1 ; \mathbf{C})$ under the condition (3.11). We suppose (3.11) in what follows. By (1) of Lemma 3.2, $P_{13}^{-1} A_{13} P_{13}$ is a diagonal matrix with different entries each other, which will be written by $D_{13}$. Any elements of matrix $B_{23}=P_{13}^{-1} A_{23} P_{13}$ are not zero. Hence by considering the adjoint actions $\left[D_{13}\left[D_{13}\left[,,,\left[D_{13}, B_{23}\right]\right]\right]\right]$ and Vandermonde determinant, it is seen matrix units $E_{i, i+1}$ and $E_{i+1, i}$ for $1 \leq i \leq n$ and $E_{i, i}$ for $1 \leq i \leq n+1$ are included into the Lie algebra generated by $D_{13}$ and $B_{23}$. This completes the proof.

The author believes that the Lie algebra $\mathscr{L} i e\left(A_{12}, A_{13}, A_{23}\right)$ generated by the residue matrices of the system (3.1) coincides with the Lie algebra Lie(PV) of the 
Picard-Vessiot group PV of (3.1).

We refer the reader for related works [2], [10], [17], [18], [19].

As for general $m$, we obtain the following.

Theorem 3.5. Fix $\ell$ as $0 \leq \ell \leq n-1$ and $\lambda_{i}+\frac{\nu}{2} \ell=0$ for $i=1, \ldots, m-2$. Then the Gauss-Manin system (2.4-5) is reducible, indeed, we have a subsystem of it with a basis

$$
\left\{\left\langle\varphi_{\left(1^{k_{1}}, \ldots,(m-1)^{k_{m-1}}\right)}\right\rangle ; 0 \leq k_{i} \leq \ell(1 \leq i \leq m-2), 0 \leq k_{m-1}\right\} .
$$

For $r=1, \ldots, m-2 ; k_{r}=\ell$,

$$
\begin{gathered}
\frac{\partial}{\partial z_{r}}\left\langle\varphi_{\left(1^{k_{1}}, \ldots, r^{\ell}, \ldots,(m-1)^{\left.k_{m-1}\right)}\right.}\right\rangle \\
=\left[\sum_{\substack{1 \leq s \leq m-1 \\
s \neq r}} \frac{\ell k_{s} \nu+\lambda_{s} \ell+\lambda_{r} k_{s}}{z_{r}-z_{s}}+\frac{\ell\left\{\lambda_{r}+\lambda_{m}+\frac{\nu}{2}(\ell-1)\right\}}{z_{r}-z_{m}}\right] \\
\quad \times\left\langle\varphi_{\left(1^{k_{1}}, \ldots, r^{\ell}, \ldots,(m-1)^{\left.k_{m-1}\right)}\right.}\right\rangle \\
+\sum_{\substack{1 \leq s \leq m-2 \\
s \neq r}} \ell\left(\lambda_{s}+\frac{\nu}{2} k_{s}\right)\left\{\frac{1}{z_{s}-z_{m}}-\frac{1}{z_{r}-z_{s}}\right\}\left\langle\varphi_{\left(\ldots, r^{\ell-1}, \ldots, s^{k, 1}, \ldots\right)}\right\rangle \\
+\ell\left(\lambda_{m-1}+\frac{\nu}{2} k_{m-1}\right)\left\{\frac{1}{z_{r}-z_{m}}-\frac{1}{z_{r}-z_{m-1}}\right\}\left\langle\varphi_{\left(\ldots, r^{\ell-1}, \ldots,(m-1)^{k_{m-1+1}}\right)}\right\rangle,
\end{gathered}
$$

for $r=1, \ldots, m-2 ; k_{r}<\ell$,

$$
\begin{aligned}
& \frac{\partial}{\partial z_{r}}\left\langle\varphi_{\left(1^{k^{k}}, \ldots,(m-1)^{\left.k_{m-1}\right)}\right.}\right\rangle= \\
& -\left(\lambda_{r}+\frac{\nu}{2} k_{r}\right) \sum_{\substack{1 \leq s \leq m-1 \\
s \neq r}} \frac{k_{s}}{z_{r}-z_{s}}\left\langle\varphi_{\left(\ldots, r^{k,+1}, \ldots, s^{k_{s}-1}, \ldots\right)}\right\rangle \\
& +\left[\sum_{\substack{1 \leq s \leq m-1 \\
s \neq r}} \frac{k_{r} k_{s} \nu+\lambda_{s} k_{r}+\lambda_{r} k_{s}}{z_{r}-z_{s}}+\frac{k_{r}\left\{\lambda_{r}+\lambda_{m}+\frac{\nu}{2}\left(k_{r}-1\right)\right\}}{z_{r}-z_{m}}\right] \\
& \times\left\langle\varphi_{\left(1^{k_{1}}, \ldots,(m-1)^{k_{m-1}}\right)}\right\rangle \\
& +\sum_{1 \leq s \leq m-2} k_{r}\left(\lambda_{s}+\frac{\nu}{2} k_{s}\right)\left\{\frac{1}{z_{r}-z_{m}}-\frac{1}{z_{r}-z_{s}}\right\}\left\langle\varphi_{\left(\ldots, r^{k,-1}, \ldots, s^{k, 1}, \ldots\right)}\right\rangle \\
& \begin{array}{l}
s \neq r \\
k_{s}<\ell
\end{array} \\
& +k_{r}\left(\lambda_{m-1}+\frac{\nu}{2} k_{m-1}\right)\left\{\frac{1}{z_{r}-z_{m}}-\frac{1}{z_{r}-z_{m-1}}\right\}\left\langle\varphi_{\left(\ldots, r^{k_{r}-1}, \ldots,(m-1)^{k_{m-1}+1}\right)}\right\rangle,
\end{aligned}
$$


for $r=m-1$,

$$
\frac{\partial}{\partial z_{m-1}}\left\langle\varphi_{\left(1^{k_{1}}, \ldots,(m-1)^{k_{m-1}}\right)}\right\rangle=
$$

$-\left(\lambda_{m-1}+\frac{\nu}{2} k_{m-1}\right) \sum_{1 \leq s \leq m-2} \frac{k_{s}}{z_{m-1}-z_{s}}\left\langle\varphi_{\left(\ldots, s^{k_{s}-1}, \ldots,(m-1)^{k_{m-1}+1}\right)}\right\rangle$

$+\left[\sum_{1 \leq s \leq m-2} \frac{k_{m-1} k_{s} \nu+\lambda_{s} k_{m-1}+\lambda_{m-1} k_{s}}{z_{m-1}-z_{s}}+\frac{k_{m-1}\left\{\lambda_{m-1}+\lambda_{m}+\frac{\nu}{2}\left(k_{m-1}-1\right)\right\}}{z_{m-1}-z_{m}}\right]$

$\times\left\langle\varphi_{\left(1^{k_{1}}, \ldots,(m-1)^{\left.k_{m-1}\right)}\right.}\right\rangle$

$$
\begin{gathered}
+\sum_{\substack{1 \leq s \leq m-2 \\
k_{s}<l}} k_{m-1}\left(\lambda_{s}+\frac{\nu}{2} k_{s}\right)\left\{\frac{1}{z_{m-1}-z_{m}}-\frac{1}{z_{m-1}-z_{s}}\right\} \\
\times\left\langle\varphi_{\left(\ldots, s^{k_{s}+1}, \ldots,(m-1)^{k_{m-1+1}}\right)}\right\rangle
\end{gathered}
$$

and for $r=m$,

$$
\begin{aligned}
& \frac{\partial}{\partial z_{m}}\left\langle\varphi_{\left(1^{k_{1}}, \ldots,(m-1)^{\left.k_{m-1}\right)}\right.}\right\rangle= \\
& \sum_{1 \leq s \leq m-1} \frac{k_{s}\left\{\lambda_{s}+\lambda_{m}+\frac{\nu}{2}\left(k_{s}-1\right)\right\}}{z_{m}-z_{s}}\left\langle\varphi_{\left(1^{k_{1}}, \ldots,(m-1)^{k_{m-1}}\right.}\right\rangle \\
& +\sum_{1 \leq s \leq m-1} \sum_{\substack{1 \leq r \leq m-2 \\
r \neq s \\
k_{r}<\ell}} \frac{k_{s}\left\{\lambda_{r}+\frac{\nu}{2}\left(k_{r}-1\right)\right\}}{z_{m}-z_{s}}\left\langle\varphi_{\left(\ldots, s^{k,-1}, \ldots, r^{k,+1}, \ldots\right)}\right\rangle \\
& +\sum_{1 \leq s \leq m-2} \frac{k_{s}\left(\lambda_{m-1}+\frac{\nu}{2} k_{m-1}\right)}{z_{m}-z_{s}}\left\langle\varphi_{\left(\ldots, s^{k_{s-1}}, \ldots,(m-1)^{k_{m-1}+1}\right)}\right\rangle .
\end{aligned}
$$

Proof. It is easily seen from the formulas in Proposition 2.1 .

Remark 3.6. The dimension of our system is the cardinarity of the set

$$
\left\{\left(k_{1}, \ldots, k_{m-1}\right) ; 0 \leq k_{i} \leq \ell(1 \leq i \leq m-2), 0 \leq k_{m-1}, k_{1}+\cdots k_{m-1}=n\right\} .
$$

For instances, it is $(\ell+1)^{m-2}$ if $n \geq(m-2) \ell$, and $\left(\begin{array}{c}n+m-2 \\ m-2\end{array}\right)$ if $\ell \geq n \geq 1$. 
Remark 3.7. In case $\ell=1$, which will be discussed in the next section, the dimension of the system is estimated as

$$
N(m, n):=1+\left(\begin{array}{c}
m-2 \\
1
\end{array}\right)+\cdots+\left(\begin{array}{c}
m-2 \\
n
\end{array}\right)
$$

which is $2^{m-2}$ if $n \geq m-2$.

\section{Spherical functions of $\mathrm{BC}$ type}

As an application of Theorem 3.5 in the case $l=1$, we derive a system of differential equations of the second order which is satisfied by the integral (0.1), which is written by $\langle 1\rangle$. It is known by Beerends-Opdam [4] and Kaneko [13] that this system characterizes some generalized hypergeometric series of matrix arguments under some conditions. Moreover, it was shown by Beerends-Opdam [4] that this generalized hypergeometric series is a special case of the hypergeometric function associated with the root system of $B C_{m}$ type defined in [11], [12].

THEOREM 4.1. For $r=1, \ldots, m-2$, put

$$
\begin{aligned}
\Delta_{r}= & \left(z_{r}-z_{m}\right)\left(z_{r}-z_{m-1}\right) \partial_{r}^{2} \\
& +\left\{\left(\frac{\nu}{2}-\lambda_{m-1}\right)\left(z_{r}-z_{m}\right)-\left(\lambda_{r}+\lambda_{m}\right)\left(z_{r}-z_{m-1}\right)\right\} \partial_{r} \\
& +n \lambda_{r}\left\{1+\lambda_{m}+\lambda_{m-1}+\frac{\nu}{2}(n-m+1)\right\} \\
& +\frac{\nu}{2}\left(z_{r}-z_{m-1}\right)\left(z_{r}-z_{m}\right) \sum_{\substack{1 \leq s \leq m-2 \\
s \neq r}} \frac{1}{z_{r}-z_{s}} \partial_{r} \\
& +\frac{\nu}{2} \sum_{\substack{1 \leq s \leq m-2 \\
s \neq r}} \frac{\left(z_{s}-z_{m-1}\right)\left(z_{m}-z_{s}\right)}{z_{r}-z_{s}} \partial_{s},
\end{aligned}
$$

where $\partial_{i}=\partial / \partial z_{i}$ and $\lambda_{r}=-\nu / 2$. Then we have $\Delta_{r}\langle 1\rangle=0$ for $r=1, \ldots, m-2$.

Proof. Fix $r(r=1, \ldots, m-2)$. By (3.12), we have

$$
\partial_{r}\left\langle\varphi_{\left((m-1)^{n}\right)}\right\rangle=\frac{n \lambda_{r}}{z_{r}-z_{m-1}}\left\{\left\langle\varphi_{\left((m-1)^{n}\right)}\right\rangle-\left\langle\varphi_{\left(r^{1},(m-1)^{n-1}\right)}\right\rangle\right\} .
$$

Hence

$$
\left\langle\varphi_{\left(r^{1},(m-1)^{n-1}\right)}\right\rangle=\left\langle\varphi_{\left((m-1)^{n}\right)}\right\rangle-\frac{z_{r}-z_{m-1}}{n \lambda_{r}} \partial_{r}\left\langle\varphi_{\left((m-1)^{n}\right)}\right\rangle
$$


On the other hand, by (3.11) we have

$$
\begin{aligned}
& =\left\{\sum_{\substack{1 \leq s \leq m-2 \\
s \neq r}} \frac{\lambda_{s}}{z_{r}-z_{s}}+\frac{(n-1)\left(\nu+\lambda_{r}\right)+\lambda_{m-1}}{z_{r}-z_{m-1}}+\frac{\lambda_{r}+\lambda_{m}}{z_{r}-z_{m}}\right\}\left\langle\varphi_{\left(r^{1},(m-1)^{n-1}\right)}\right\rangle \\
& +\sum_{\substack{1 \leq s \leq m-2 \\
s \neq r}} \lambda_{s}\left\{\frac{1}{z_{r}-z_{m}}-\frac{1}{z_{r}-z_{s}}\right\}\left\langle\varphi_{\left(s^{1},(m-1)^{n-1}\right)}\right\rangle \\
& +\left(\lambda_{m-1}+\frac{\nu}{2}(n-1)\right)\left\{\frac{1}{z_{r}-z_{m}}-\frac{1}{z_{r}-z_{m-1}}\right\}\left\langle\varphi_{\left((m-1)^{n}\right)}\right\rangle .
\end{aligned}
$$

By substituting (4.3) into both sides of (4.4), we finally obtain

$$
\begin{gathered}
\left\{\left(z_{r}-z_{m}\right)\left(z_{r}-z_{m-1}\right) \partial_{r}^{2}+\left\{\left(1+\frac{\nu}{2}-\lambda_{m-1}\right)\left(z_{r}-z_{m}\right)-\left(\lambda_{r}+\lambda_{m}\right)\left(z_{r}-z_{m-1}\right)\right\} \partial_{r}\right. \\
+n \lambda_{r}\left\{\lambda_{m}+\lambda_{m-1}+\frac{\nu}{2}(n-m+1)\right\}+\frac{\nu}{2}\left(z_{r}-z_{m-1}\right)\left(z_{r}-z_{m}\right) \sum_{\substack{1 \leq s \leq m-2 \\
s \neq r}} \frac{1}{z_{r}-z_{s}} \partial_{r} \\
\left.+\frac{\nu}{2} \sum_{\substack{1 \leq s \leq m-2 \\
s \neq r}} \frac{\left(z_{s}-z_{m-1}\right)\left(z_{m}-z_{s}\right)}{z_{r}-z_{s}} \partial_{r}\right\}\left\langle\varphi_{\left((m-1)^{n}\right)}\right\rangle=0 .
\end{gathered}
$$

The equation (4.1) is given by substituting $\lambda_{m-1}+1$ for $\lambda_{m-1}$ in (4.5).

Remark 4.2. If we put $z_{m}=0$ and $z_{m-1}=1$ in (4.1), then the operators $\Delta_{r}(1 \leq r \leq m-2)$ turn out to be

$$
\begin{aligned}
\Delta_{r}=z_{r}\left(1-z_{r}\right) \partial_{r}^{2}+\left\{\left(\frac{\nu}{2}-\lambda_{m}\right)-\left(\nu-\lambda_{m}-\lambda_{m-1}\right) z_{r}\right\} \partial_{r} \\
+\frac{n \nu}{2}\left\{1+\lambda_{m}+\lambda_{m-1}+\frac{\nu}{2}(n-m+1)\right\} \\
+\frac{\nu}{2} z_{r}\left(1-z_{r}\right) \sum_{\substack{1 \leq s \leq m-2 \\
s \neq r}} \frac{1}{z_{r}-z_{s}} \partial_{r} \\
\quad-\frac{\nu}{2} \underset{\substack{1 \leq s \leq m-2 \\
s \neq r}}{\sum_{s}\left(1-z_{s}\right)} \partial_{s} .
\end{aligned}
$$

Remark 4.3. By taking various $\Gamma \in H_{n}\left(X, S_{\omega}^{*}\right)^{S_{n}}$, we can construct $N(m, n)$ solutions of (4.6) expressed by the integral (0.1) (see Remark 3.7). Notice 
that

$$
\left\{\begin{array}{l}
N(m, n)=2^{m-2}, \text { when } n \geq m-2, \\
N(m, n)<2^{m-2}, \text { when } n<m-2 .
\end{array}\right.
$$

In [13], Kaneko states without proof that the rank of the system (4.6) is $2^{m-2}$ (the author has been told by him that he has proved this fact). The author does not know if the rank of the system (4.6) equals to $N(m, n)$ when $n<m-2$.

Acknowledgements. The author thanks to Professors Kazuhiko Aomoto and Masatoshi Noumi for beneficial discussions, and to Professor Ryoshi Hotta for a useful comment. The author also expresses his sincere gratitude to Professor Masaaki Yoshida for stimulating discussions and careful reading the manuscript.

\section{REFERENCES}

[1] K. Aomoto, Un théorème du type de Matsushima-Murakami concernant l'intégrale des fonctions multiformes, J. Math. Pures Appl., 52 (1973), 1-11.

[2] - Fonctions hyperlogarithmiques et groupes de monodromie unipotents, J. Fac. Sci. Univ. Tokyo, 25 (1978), 149-156.

[ 3 ] - Gauss-Manin connection of integral of difference products, J. Math. Soc. Japan, 39 (1987), 191-208.

[ 4 ] R. J. Beerends and E. M. Opdam, Certain hypergeometric series related to the root system BC, preprint 1990.

[ 5 ] E. Brieskorn, Sur les groupes de tresses. In: Sém. Bourbaki, Lecture Notes in Math., 317, pp. 21-44, Springer 1973.

[6] E. Date, M. Jimbo, A. Matsuo and T. Miwa, Hypergeometric type integrals and the $s l(2, \mathbf{C})$ Knizhnik-Zamolodchikov equation, in "Yang-Baxter equations, conformal invariance and integrability in statistical mechanics and field theory", World Scientific (1989).

[ 7 ] P. Deligne, Equations Differentielles à Points Singulier Regulier, Lecture Notes in Math., 163, Springer 1970.

[ 8 ] V.S. Dotsenko and V. A. Fateev, Conformal algebra and multipoint correlation functions in 2D statistical models, Nucl. Phys., B240 (1984), 312-348.

[ 9 ] H. Esnault, V. Schechtman and E. Viehweg, Cohomology of local system on the complement of hyperplanes, Invent. Math., 109 (1992), 557-561. Errata. ibid., 112 (1993), 447.

[10] R.M. Hain, On a generalization of Hilbert's 21st problem, Ann. Sci. École Norm. Sup., 19 (1986), 609-627.

[11] G.J. Heckman and E. M. Opdam, Root systems and hypergeometric functions I, Comp. Math., 64 (1987), 329-352.

[12] G.J. Heckman, Root systems and hypergeometric functions II, Comp. Math., 64 (1987),353-374.

[13] J. Kaneko, Selberg integrals and hypergeometric functions associated with Jack polynomials, SIAM J. Math. Anal., 24 (1993), 1086-1110. 
[14] M. Kita and M. Noumi, On the structure of cohomology groups attached to certain many valued analytic functions, Japan. J. Math., 9 (1983), 113-157.

[15] M. Kita, On hypergeometric functions in several variables 1 . New integral representations of Euler type, Japan. J. Math., 18 (1992), 25-74.

[16] T. Kohno, Homology of a local system on the complement of hyperplanes Proc. Japan Acad., 62, Ser. A (1986), 144-147.

[17] - On the holonomy Lie algebra and the nilpotent completion of the fundamental group of the complement of hypersurfaces, Nagoya Math. J., 92 (1983), 21-37.

[18] - Hecke algebra representations of braid groups and classical Yang-Baxter equations, Adv. Stud. Pure Math., 16 (1988), 255-269.

[19] I.A. Lappo-Danilevsky, Mémoires sur la théorie des systèmes des équations différentielles linéaires, reprint, Chelsea, New York, 1953.

[20] T. Sasaki, Picard-Vessiot group of Appel's system of hypergeometric differential equations and infiniteness of monodromy group, preprint 1979.

[21] V.V. Schechtman and A.N. Varchenko, Arrangements of hyperplanes and Lie algebra homology, Invent. Math., 106 (1991), 139-194.

[22] A. Selberg, Bemerkninger om et Mulitiplet Integral, Norsk Mat. Tidsskrift, 26 (1944), $71-78$

[23] J.P. Serre, Géométrie algébrique et géométrie analytique, Ann. Inst. Fourie, 6 (1956), $1-42$.

[24] K. Takano, and E. Bannai, A global study of Jordan-Pochhammer differential equations, Funkcial. Ekvak., 19 (1976), 85-99.

[25] A. Tsuchiya and Y. Kanie, Fock space representations of the Virasoro algebra -Intertwining operators, Publ. RIMS. Kyoto Univ., 22 (1986), 259-327.

[26] - Vertex operators in conformal field theory on $\mathbf{P}^{1}$ and monodromy representations of braid group, Adv. Stud. Pure Math., 16 (1988), 297-372.

Department of Mathematics

Faculty of Science

Kyushu University

Hakozaki, Fukuoka 812, Japan 